\title{
Delegating Responsibility from Clinicians to Nonprofessional Personnel: The Example of Hypertension Control
}

\author{
David Margolius, MD, Jennifer Wong, BA, Matthew L. Goldman, MS, \\ James Rouse-Iniguez, MA, and Thomas Bodenheimer, MD, MPH
}

Background: Involving nonclinician personnel in the treatment of hypertension may provide a solution to improve blood pressure control; however, this team-based approach cannot be implemented without first determining clinicians' willingness to delegate patient care to nonclinician team members. This study explores clinicians' perspectives on working with nonclinicians trained as "health coaches" to address medication adherence and lack of medication intensification among low-income patients with uncontrolled hypertension.

Methods: We used a qualitative research approach to determine clinicians' opinions on the Treat-toTarget study, an intervention to improve blood pressure control. We conducted focus groups with clinicians who practice family medicine in a safety net clinic. Transcripts were analyzed using thematic content analysis.

Results: Seven overarching themes emerged: (1) Clinicians support the delegation of functions to health coaches; (2) clinicians like the high frequency of coach-patient interactions; (3) clinicians feel that health coaching assists medication adherence; (4) clinicians have varying views on home titration; (5) coach-clinician communication is necessary for successful delegation; (6) coaching helps clinicians understand their patients' barriers to hypertension control; and (7) clinicians would like health coaching to continue on a permanent basis.

Conclusion: Clinicians appreciate the presence of nonclinicians on the primary care team. In the coming era of primary care clinician shortage, clinicians can be supportive of nonprofessional team members assisting with the care of patients with hypertension. (J Am Board Fam Med 2012;25:

209-215.)

Keywords: Health Care Team, Hypertension, Primary Health Care

Adult primary care faces a dilemma. On the one hand, the worsening shortage of adult primary care

This article was externally peer reviewed.

Submitted 21 November 2010; revised 27 February 2011; accepted 2 March 2011.

From the Department of Family and Community Medicine, University of California, San Francisco (DM, JW, JR-I, TB); the Warren Alpert Medical School of Brown University, Providence, Rhode Island (DM); and the University of California Berkeley-University of California San Francisco Joint Medical Program, Berkeley, CA (MG).

Funding: This research was funded by the Kaiser Permanente Community Benefits Program Northern California Region (grant reference number 20602348).

Conflict of interest: none declared.

Corresponding author: Thomas Bodenheimer, Department of Family and Community Medicine, University of California, San Francisco, Bldg 80-83, San Francisco General Hospital, 995 Potrero Ave, San Francisco, CA 94110 (E-mail: TBodenheimer@fcm.ucsf.edu). physicians imperils access to care for millions of Americans. ${ }^{1}$ On the other hand, panel sizes are too large for adult primary care physicians to handle. It would take almost 18 hours to provide high-quality preventive and chronic care to a panel of 2300 patients, which is the average primary care physician panel size. ${ }^{2}$ The dilemma arises in the need to increase panel size to make up for the adult primary care physician shortage but the impossibility of doing so because of the amount of time it takes to care for larger panels.

One solution lies in delegating less complex activities from physicians to other members of the primary care team so that the team, not solely the physician, becomes responsible for the health of a large panel of patients. ${ }^{3}$ Such delegation would 
traditionally involve nurse practitioners (NPs), physician assistants (PAs), registered nurses (RNs), and pharmacists. However, NPs and PAs are needed to do physician-level work to address the physician shortage, whereas RNs and pharmacists are not commonly found in primary care practices because of their relatively high salaries and the lack of reimbursement for their services by most payers. Thus, delegation needs to involve nonprofessional personnel.

In the study described here, substantial portions of the care of patients with poorly controlled hypertension were delegated to health coaches-in this case, personnel who were college educated but had no professional medical training. Health coaching consists of making sure that patients are knowledgeable about their disease and their medications, ensuring that patients understand and agree with their physician's care plan, assisting patients to make healthy behavior choices, and working with patients on medication adherence. ${ }^{4,5}$

\section{Background}

Hypertension affects 65 million people in the United States. Fifty percent have achieved blood pressure levels less than 140/90 $\mathrm{mm} \mathrm{Hg}$, leaving more than 32 million Americans at risk for the serious consequences of uncontrolled hypertension. ${ }^{6}$ Universal treatment of hypertension could avoid an estimated 46,000 deaths per year among persons younger than 80 years of age. ${ }^{7}$

Two important reasons why hypertension is difficult to control are poor medication adherence and "clinical inertia." Adherence rates among patients with hypertension vary depending on the measurement method used, and rates are significantly lower among minorities ${ }^{8}$; a clinic caring for vulnerable populations found only $36 \%$ good adherence among patients with hypertension. ${ }^{9}$ Lower adherence is associated with poor blood pressure control, disease progression, disability, and death. ${ }^{10}$

Clinicians often fail to intensify medications during visits when blood pressure recordings are high. ${ }^{11}$ This phenomenon, called "clinical inertia," is related to competing priorities: visits in which too many agenda items must be addressed in the time available. ${ }^{12}$ In one study, $83 \%$ of ambulatory visits during which patients with hypertension had elevated readings were associated with either poor adherence or the failure of clinicians to intensify medications appropriately. ${ }^{13}$
The Department of Family and Community Medicine, University of California, San Francisco (UCSF) initiated a Treat to Target (TTT) study in 2008. The study was conducted at San Francisco General Hospital's Family Health Center, a teaching clinic for UCSF family medicine residents. TTT is a 3-pronged hypertension innovation that addresses medication adherence and clinical inertia and tests whether important aspects of hypertension management can be delegated from physicians to nonprofessional health coaches. This article examines the perspectives of Family Health Center clinicians (physicians and NPs) who participated in the TTT study. As noted earlier, a key goal of this study was to determine how clinicians viewed the delegation of important aspects of hypertension management to nonprofessional team members. The delegation of clinical responsibility in an era of large panels and growing physician shortage will take place only if clinicians are comfortable with the involvement of nonprofessional team members.

\section{Methods}

\section{Overview of the Treat to Target Study}

English-, Spanish-, Cantonese-, and Vietnamesespeaking patients were recruited from the Family Health Center and were randomly assigned to the control group, which received regular phone calls, or to the active group, which received regular phone calls plus coach-assisted home titration.

\section{The Intervention}

First, patients with high blood pressure were given a home blood-pressure monitor and were trained in its use. Second, patients received regular phone calls from a health coach who reinforced blood pressure goals and counseled them about diet, exercise, and medication adherence. Third, using physician-approved treatment protocols or standing orders, patients and health coaches could jointly decide by telephone to intensify antihypertensive medications. This process of coach-assisted home titration could reduce the need for physician visits. If medication doses were changed, the health coach notified the physician.

All patients were given a logbook and an Omron HEM-711AC blood pressure cuff (Omron Healthcare, Inc., Palatine, IL) and were trained in its use by a language-concordant health coach. The intervention lasted for 6 months. Inclusion 
criteria and details of the recruitment process can be found in the TTT study protocol. ${ }^{14}$ Ten UCSF employees and volunteers, 8 of whom had no formal clinical education, were trained as health coaches. Coaches attended training about health coaching techniques, hypertension pathophysiology, and treatment. Health coaches were assigned 5 to 30 patients each. Phone encounters involved documenting blood pressure and heart rate, medication reconciliation, and self-management support. If blood pressure remained above target for "active" patients, medications were titrated using clinician-approved care plans. $\mathrm{Pa}$ tient encounters and medication adjustments were logged into a database.

\section{Setting and Participants}

Focus group interviews were conducted at the Family Health Center. Clinicians with at least one patient enrolled in the study were eligible to participate, and 10 of the 26 eligible clinicians participated. The participants had from 2 to 25 patients enrolled in the study. The 10 focus group participants were recruited as a convenience sample ${ }^{15}$; however, there was a purposive component to the sampling in 2 ways. First, the participants in the focus groups were the providers for $32 \%$ of all the patients in the study, so the small number of participants in the focus groups underestimates the major role of those providers in the study. Second, the proportion of focus group participants who were faculty physicians, resident physicians, or NPs was similar to the proportion of those from those 3 provider categories who participated in the study, which is in line with establishing a quota sampling frame. The qualitative study was approved by the UCSF Committee on Human Research and all participants provided written, informed consent.

\section{Data Collection}

Two focus group interviews were conducted in April $2010(\mathrm{n}=6$ and $\mathrm{n}=4)$ according to methods outlined by Schensul. ${ }^{16}$ The same open-ended questions were asked at both sessions. Questions encouraged participants to recount specific experiences when the TTT study may have affected their management of hypertension and to describe interactions with their health coaches. Both focus group interviews lasted between 45 and 60 minutes, and they were audio recorded and transcribed verbatim.

\section{Data Analysis}

The analysis team included one physician with public health training, a medical student with health coaching experience, a master's level investigator, and a research assistant. Using methods suggested by Miles and Huberman, ${ }^{15}$ all team members independently read both transcripts and identified 3 to 10 descriptive codes to organize the themes within the data. The unit of analysis was the focus group, and the analysis team took into account within-group interactions among the participants and between-group similarities and differences. Triangulation was achieved through an iterative and collaborative process among team members to reach consensus about this preliminary codebook and then to group the descriptive codes into inferential categories. The outcomes of this thematic content analysis reflected emerging trends in the data, as evident from the prevalence of the following themes and the reiteration of particular points of view.

\section{Results \\ Participant Characteristics}

Focus group participants included 5 faculty members, 4 residents, and one NP. All clinicians were trained in family medicine. The group was comprised of 7 women and 3 men. Time in practice ranged from 2 to 43 years, with a median of 8 years.

\section{Themes}

Seven main themes were identified.

\section{Clinicians Support the Delegation of Functions to Health Coaches}

Focus group participants were unanimously positive about the experience of delegating the coaching function. Multiple clinicians felt that health coaches assisted patients to understand their readings and how those readings compared with their target blood pressure, and the physicians expressed that the coaching led to increased interest by their patients in managing their hypertension. One focus group participant described a patient with uncontrolled blood pressure who had no interest in controlling her blood pressure for years; when she began to receive health coaching, she became motivated to bring her blood pressure under control.

"And this was completely attributable to being involved in TTT and having her health coach, if that's the 
right word, calling her at home and checking on her readings and having her see the changes that happened as she changed her medications." (Resident)

Another participant added:

"When I was approached about adding [a patient] to TTT, I was very skeptical about whether it would work... I was really surprised when he responded very positively, took the blood pressure cuff home, started taking his blood pressure regularly... I just saw him last week and we no longer have to fight about his blood pressure medication anymore. His blood pressure was terrific. He's still taking his blood pressures at home. It was really quite amazing to me." (Faculty)

\section{Clinicians Liked the High Frequency of Coach-Patient Interactions}

Clinicians expressed that delegation of responsibility to health coaches allowed frequent contact between health coaches and patients. Clinicians acknowledged that having a health coach who made frequent contact with the patient was a critical component to the success of the intervention.

"There were a couple times because the... bealth coaches were in direct contact by phone and some of them had developed good relationships, there was much better adherence in the self-monitoring at bome." (Faculty)

Clinicians admitted they lack time to follow up with patients regularly after appointments and appreciated that their patients had someone with whom they had direct contact. This point was especially true for patients with psychosocial and other challenges who require more attention. One clinician acknowledged that these patients benefited in ways beyond improved blood pressure control.

"I think it was really great for them to be able to bave somebody to call and talk to about their blood pressure. It made them feel that there was someone really responsive to them in a way that I can't always be in their medical care." (Faculty)

Clinicians also found health coaching valuable for helping patients with lifestyle changes.

"The health coaches also worked with my patients on cutting down on sodium, especially my Asian patients. They worked on using less soy sauce and prepared noodles...Their blood pressure came into control, I think, more from that than from anything else." (Faculty)

\section{Clinicians Feel That Health Coaching Assists Medication Adberence}

Medication adherence was an important issue for clinicians when it comes to hypertension control. Clinicians faced language and cultural barriers in patients' ability to take medications correctly. Providers repeatedly mentioned that delegating responsibility to the coaches helped a great deal with medication adherence.

"I sort of felt that she was taking her medications when she had a bealth coach. She came in and all lof a] sudden her blood pressures were great and I was like, 'oh, it turns out she was actually on good medicine but she just wasn't taking it.' I think a lot of it is confusion on her part about what to take and how to take it." (Faculty)

Clinicians thought it was helpful to have a second person make contact with patients, especially between visits. They agreed that health coaches in some instances were able to expose situations of nonadherence, even when clinicians thought they had reconciled medications with the patient during the visit. One resident shared a story about when her patient revealed to the health coach that she was unable to pick up the medication from the pharmacy.

"The bealth coach was the one who found that they badn't been taking the medicine because they hadn't been able to get it from the pharmacy and were embarrassed to say that they hadn't been able to get it." (Resident)

\section{Clinicians Have Varying Views On Home Titration}

A dichotomy in opinions emerged regarding coachassisted home titration. Younger clinicians (residents) liked home titration more than older clinicians (faculty). Some clinicians found home titration beneficial because patients would not have to come into clinic for a blood pressure follow-up, especially when there were no open appointments.

"I think it still worked very well to engage patients in self-monitoring and off-loading a little bit of having to make a whole visit just for a blood pressure check-in and titration." (Resident)

"I think, just as residents, it was helpful because we often can't see folks as frequently as we want. It was belpful in that sense that they could make adjustments without having to wait 1 month or 3 months to get back to see us." (Resident)

However, older clinicians preferred to do medication titration themselves and viewed it as secondary to health coaching. Some clinicians expressed confusion 
over the home titration component because of miscommunication with the pharmacy or health coach after a medication was adjusted. One older clinician was concerned that the coach did not understand the antihypertensive medications enough to be changing them.

"I like the idea of having those protocols and maximizing efficacy of our medication regimen, but when it is so often around adherence and other issues it just feels like it's not that important to go for like [benazepril] from 10 to 20. It's better to sort of understand the potential for bad effects of that and deal with adherence and let us deal with the medication adjustment." (Faculty)

\section{Coach-Clinician Communication Is Necessary for Successful Delegation}

Communication between health coaches and clinicians was viewed as an important aspect of delegating the coaching functions. Most clinicians thought that E-mail communication from health coaches was helpful, and they valued the fact that the coaches acted as intermediaries between the clinician and the patient.

"The communication was very smooth between the patient, his health coach, and me. His bealth coach would usually send me an E-mail if issues came up." (Resident)

Some health coaches became advocates for their patients by relaying messages not relevant to blood pressures.

"The bealth coaches... would convey that. . they ran out of these medications or...she's been complaining of bright red blood. . . been passing these large stools,... she didn't know if you knew about this, she didn't know where to go." (Faculty)

There were some challenges to coach-clinician communication. Some clinicians felt protective of their patients and initially felt uncomfortable sharing care, but eventually warmed to working with the health coaches. Other clinicians found that coaching without home titration was difficult.

"Ideally [it] sounds like a great thing that somebody would be E-mailing me and telling me things ... but I just don't have any time outside of clinic ... to call somebody in between a visit about it." (Resident)

\section{Coaching Helps Clinicians Understand Their Patients' Barriers to Hypertension Control}

Clinicians felt that the teamwork involved in the coach-clinician relationship helped them distin- guish patients who were willing to take their blood pressure and follow medication regimens from patients who were not willing or able to do so.

"I think it actually helped me separate who was really actively addressing self-management of their bealth or ... wasn't in a place where they were really going to participate." (Resident)

Clinicians noticed 2 types of outcomes after the coaching ended: either the patients continued to check their blood pressure and maintain good control, or blood pressure levels jumped back up to baseline levels and all signs of behavioral change vanished. The ending of the TTT intervention helped providers figure out which of their patients had the biggest problems with adhering to their treatment plan.

"It was almost like a diagnostic tool; a little bit like, 'why are their blood pressures out of control?'” (Faculty)

\section{Clinicians Would Like Health Coaching To Continue On a Permanent Basis}

Concern was expressed that phone calls between the patient and health coach were being discontinued. A few clinicians mentioned that some patients' blood pressures jumped back up after the study ended. Questions were raised about the sustainability of the behavior changes.

"For her it was really clear that the second someone stopped calling, any behavioral change that was made was gone." (Faculty)

"The beauty of this was knowing that there was someone out there who was checking in-which was really important-helping to measure and advocate. And that was huge. And I'm really sorry that it's over because now we're back to where we were." (NP)

Many of the clinicians realized the importance of being able to delegate responsibility to clinic staff to provide self-management support and follow-up to patients on a permanent basis.

\section{Discussion}

Because $79 \%$ of physician visits for hypertension take place in primary care, the shortage of primary care physicians may undermine the capacity of the health care system to maintain and improve the management of hypertension. ${ }^{17}$ The study reported here suggests a new approach to hypertension management that asks less of overburdened physicians by delegating important functions to nonprofessional health coaches. Delegating impor- 
tant patient-care services to other team members will only succeed if clinicians are in agreement with this approach. The results of the clinician focus groups demonstrate that clinicians in an academic family medicine practice support team-based care for patients with hypertension.

Focus group participants agreed that the addition of a health coach to the team improves adherence to hypertension treatment plans and that frequent contacts with the health coach facilitates lifestyle modifications on the part of patients. All clinicians seemed eager to share the work of promoting medication adherence and lifestyle medications with nonclinicians.

Less consensus surrounded home titration. Older faculty clinicians felt that health coaching to titrate medications is not as important to improving blood pressure as is health coaching to promote adherence. Interestingly, whereas older clinicians had difficulty delegating home titration to health coaches, younger resident clinicians were comfortable with this delegation of functions. Younger clinicians felt that delegating the responsibility of titrating antihypertensive medication would allow more prompt management of their patients' hypertension, given the paucity of available appointments in their schedules.

Studies have been published about the delegation of responsibilities to pharmacists and nurses to improve blood pressure control. ${ }^{18-20}$ The study reported here is unique because it evaluates the role of nonlicensed personnel in the management of hypertension. We were unable to find previous studies regarding delegation of responsibilities to nonprofessional personnel for hypertension. A few studies examine the delegation of some aspects of chronic care management to medical assistants. In a US academic family medicine clinic, teamlets of medical assistants and nonprofessional health workers paired with clinicians were associated with improved cardiovascular risk reduction outcomes when contrasted with a comparison group. ${ }^{21}$ In a German study, medical assistants helping physicians with the management of depression reduced depression symptoms compared with usual care, and the medical assistants were satisfied with assuming this new role. ${ }^{22}$ In those 2 studies, the physicians' attitudes toward delegation were not examined. Physician participants in focus groups in Germany did express positive attitudes toward delegating to medical assistants the responsibility of monitoring heart failure patients through phone calls and home visits. ${ }^{23}$

One limitation of this qualitative study was the small sample size compared with the number of clinicians who had patients enrolled in the TTT study. Nevertheless, given the range in years of practice for the focus group participants, we feel that their acceptance of the intervention, especially their appreciation of the health coaches, was representative of the entire group of eligible clinicians.

\section{Conclusion}

Health coaching is a departure from the traditional model of care because it partially delegates responsibility for patient care to nonprofessional staff. Sustainability of this or similar interventions will require a redesign of primary care practice. The clinicians we interviewed were accepting of many components of the team-based care approach. In an era of primary care clinician shortage, reorganizing practices to expand the roles of ancillary staff to include health coaching may help to improve blood pressure control without placing new demands on overwhelmed primary care clinicians.

The authors would like to acknowledge Guillermo Padilla, Victoria Ngo, Maria Vivas, Jorge Palacios, Kelsey Laird, Ariel Postone, Sandi Ma, Mitchell Hawkins, Heather Bennett, Rachel Berry-Millett, Gloria Garcia, and Sachini Bandara for their coaching of patients in the TTT study. We wish to thank the Omron company for donating blood pressure monitoring devices.

\section{References}

1. Bodenheimer T, Pham HH. Primary care: current problems and proposed solutions. Health Aff 2010; 29:799-805.

2. Yarnall KSH, Ostbye T, KrauseKM, Pollak KI, Gradison M, Michener JL. Family physicians as team leaders: "time" to share the care. Prev Chronic Dis 2009;6:1-6.

3. Margolius D, Bodenheimer T. Transforming primary care: from past practice to the practice of the future. Health Aff 2010;29:779-84.

4. Chen E, Thom DH, Hessler DM, et al. Using the teamlet model to improve chronic care in an academic primary care practice. J Gen Intern Med 2010; 25(Suppl 4):610-4.

5. Bennett HD, Coleman EA, Parry C, Bodenheimer $\mathrm{T}$, Chen EH. Health coaching for patients with chronic disease. Fam Pract Manag 2010; Sept-Oct: 24-9. 
6. Egan BM, Zhao Y, Axon RN. US trends in prevalence, awareness, treatment, and control of hypertension, 1988-2008. JAMA 2010;303:2043-50.

7. Farley TA, Dalal MA, Mostashari F, Frieden TR. Deaths preventable in the U. S. by improvements in use of clinical preventive services. Am J Prev Med 2010;38:600-9.

8. Krousel-Wood MA, Muntner P, Islam T, Morisky DE, Webber LS. Barriers to and determinants of medication adherence in hypertension management: perspective of the cohort study of medication adherence among older adults. Med Clin North Am 2009; 93:753-69.

9. Bosworth HB, Powers B, Grubber JM, et al. Racial differences in blood pressure control: potential explanatory factors. J Gen Intern Med 2008;23:692-8.

10. Osterberg L, Blaschke T. Adherence to Medication. N Engl J Med 2005;353:487-97.

11. Phillips LS, Branch WT, Cook CB, et al. Clinical inertia. Ann Intern Med 2001;135:825-34.

12. Safford MM, Shewchuk R, Qu H, et al. Reasons for not intensifying medications: differentiating "clinical inertia" from appropriate care. J Gen Intern Med 2007;22:1648-55.

13. Heisler M, Hogan MM, Hofer TP, Schmittdiel JA, Pladevall M, Kerr EA. When more is not better: treatment intensification among hypertensive patients with poor medication adherence. Circulation 2008;117:2884-92.

14. Bennett H, Laird K, Margolius D, Ngo V, Thom $\mathrm{DH}$, Bodenheimer $\mathrm{T}$. The effectiveness of health coaching, home blood pressure monitoring, and home-titration in controlling hypertension among low-income patients: protocol for a randomized controlled trial. BMC Public Health 2009;9:456.
15. Miles MB, Huberman AM. Focusing and bounding the collection of data: the substantive start. In: Qualitative Data Analysis: An Expanded Sourcebook. 2nd edition. Thousand Oaks: Sage, 1994:50-89.

16. Schensul JJ. Focused group interviews. In: Schenul JJ, LeCompte MD, Nastasi BK, Borgatti SP, eds. Enhanced Ethnographic Methods. Walnut Creek, CA: AltaMira Press; 1999:51-114.

17. Fang J, Keenan NL, Ayala C. Health care services provided during physician office visits for hypertension: differences by specialty. J Clin Hypertens 2010; 12:89-95.

18. Mehos BM, Saseen JJ, MacLaughlin EJ. Effect of pharmacist intervention and initiation of home blood pressure monitoring in patients with uncontrolled hypertension. Pharmacotherapy 2000;20: 1384-9.

19. Hunt JS, Siemienczuk J, Pape G, et al. A randomized controlled trial of team-based care: impact of physician-pharmacist collaboration on uncontrolled hypertension. J Gen Intern Med 2008;23:1966-72.

20. Edelman D, Fredrickson SK, Melnyk SD, et al. Medical clinics versus usual care for patients with both diabetes and hypertension: a randomized trial. Ann Intern Med 2010;152:689-96.

21. Chen EH, Thom DH, Hessler DM, et al. Using the Teamlet Model to improve chronic care in an academic primary care practice. J Gen Intern Med 2010; 25(Suppl 4):S610-4.

22. Gensichen J, Jaeger C, Peitz M et al. Health care assistants in primary care depression management. Ann Fam Med 2009;7:513-9.

23. Peters-Klimm F, Olbort R, Campbell S, et al. Physicians' view of primary care-based case management for patients with heart failure. Intern J Qual Health Care 2009;21:363-71. 